\title{
Stereoscopic observations of the giant hard X-ray/gamma-ray solar flare on 1991 June 30 at 0255 UT
}

\author{
G. Trottet ${ }^{1}$, R. A. Schwartz ${ }^{2}$, K. Hurley ${ }^{3}$, J. M. McTiernan ${ }^{3}$, S. R. Kane ${ }^{3}$, and N. Vilmer ${ }^{1}$ \\ 1 LESIA, Observatoire de Paris, Section de Meudon, 92195 Meudon, France \\ 2 NASA/Goddard Space Flight Center, Code 682, Greenbelt, MD 2077, USA \\ 3 Space Sciences Laboratory, University of California, Berkeley, CA 94720, USA
}

Received 19 November 2002 / Accepted 19 March 2003

\begin{abstract}
The hard X-ray/gamma-ray (HXR/GR) impulsive burst on 1991 June 30 ( 0255 UT) was associated with a flare which occured between $2^{\circ}$ and $12^{\circ}$ behind the east limb of the Sun. The partially occulted HXR/GR emission from this flare was detected at up to $100 \mathrm{MeV}$ by three instruments on Earth-orbiting spacecraft: the Burst and Transient Source Experiment (BATSE) and the Energetic Gamma-Ray Experiment (EGRET) on CGRO and by the Payload for High Energy Burst Spectroscopy (PHEBUS) on GRANAT. As seen from the two spacecraft in Earth orbit, the size of the burst corresponds to that of a moderate electron-dominated GR event (Dingus et al. 1994; Vilmer et al. 1999). However, this event is one of the giant flares reported by Kane et al. (1995). It was observed by the Solar X-ray/Cosmic Gamma-Ray Burst Experiment (GRB) on Ulysses, located $135^{\circ}$ east of the Earth-Sun line. GRB measured the total $>28 \mathrm{keV}$ HXR emission from the flare. In this paper we combine HXR observations by GRB and BATSE in order to determine the time evolution of the power-law index $\gamma$ of the photon spectrum of the partially occulted HXR emission seen by BATSE and of the fraction $R$ of the partially occulted to the total $>28 \mathrm{keV}$ emission. $\gamma$ decreased from $\sim 5.4$ to $\sim 2.6$ and $R$ varied from $\sim 20 \%$ at the beginning of the event down to $<1 \%$ at its maximum. These results indicate that the spatial distribution of the HXR sources was complex, and evolved in the course of the event. While the HXR emission detected by GRB was almost entirely produced at the footpoints of loops by thick-target interactions, a fraction of the HXR emission seen by BATSE likely originated in the unocculted, low density, portion of the HXR emitting loops. The data also show that a small fraction ( 10\%) of the HXR emission detected by BATSE in Earth orbit was radiated by a thick-target source on the visible disk.
\end{abstract}

Key words. Sun: activity - Sun: flares - Sun: particle emission - Sun: X-rays, gamma-rays

\section{Introduction}

The most direct information on the characteristics of energetic electrons at the Sun is provided by the $\sim 20 \mathrm{keV}-100 \mathrm{MeV}$ bremsstrahlung continuum produced by these electrons as they interact with the ambient solar atmosphere. Observations of HXR emitting sources made with high spatial, spectral, and temporal resolution are therefore essential for understanding the acceleration and propagation of these electrons in solar flares. Over the last two decades HXR imaging observations have been obtained with instruments on board the Solar Maximum Mission (SMM), Hinotori and Yohkoh. Briefly stated, the spatial distribution of HXR sources was found to vary from flare-to-flare and to evolve with time during some flares. For example:

- There are simple configurations where the HXR emission originates at the footpoints of a single magnetic loop or loop system with lengths of a few times $10^{4} \mathrm{~km}$ (Duijveman et al. 1982; Sakao 1994). For a few flares, a faint coronal

Send offprint requests to: G. Trottet,

e-mail: Gerard.trottet@obspm.fr source was also observed near or above the top of the loop (Masuda et al. 1994, 1995).

- More complex and time varying distributions of HXR sources have also been revealed e.g.: (i) Sakao et al. (1992) reported observations of one flare suggesting that successive HXR peaks correspond to energy release in different loops; (ii) combined HXR and microwave imaging observations of a few flares (Nakajima et al. 1985) have shown that these emissions originated both in active region loops of a few times $10^{4} \mathrm{~km}$ length and in much larger scale loops (a few times $10^{5} \mathrm{~km}$ ): HXR emission was detected at large scale loop footpoints remote from the flare site; (iii) during a two-ribbon-flare, it was found (Masuda et al. 2001) that the HXR emission was produced at the footpoints of a large arcade of active region loops (width $\sim 3 \times 10^{4} \mathrm{~km}$, length $\sim 10^{5} \mathrm{~km}$ ) connecting the flare ribbons: at the beginning of the event the HXR emission originated predominantly from loops in the leading part of the magnetic arcade but later on it originated from loops in its trailing part; and (iv) recent observations of an X-class flare close to the solar limb by the Reuven Ramaty High-Energy Solar Spectroscopic 
Imager (RHESSI) have provided evidence that the spatial distribution of the HXR sources may vary during a given event (Gallagher et al. 2002).

As noticed by Kane (1987), there may be some ambiguity in determining the relative brightness of the different HXR emitting sources from imaging observations since the observed brightness of an HXR source is in reality the integrated brightness along the line of sight. Observations obtained with imaging instruments on board SMM, Hinotori and Yohkoh suffered from further limitations such as: an energy range restricted to below 30, 40, and $100 \mathrm{keV}$ respectively and a dynamic range which does not allow us to detect sources with brightnesses below a few tens of percent of the brightest source. Such limitations will be partly removed by RHESSI, which was designed to provide images up to $\mathrm{MeV}$ energies and, in favorable circumstances, with a dynamic range of 100:1 at lower energies (Hurford et al. 2002). Thus, only in the case where the associated flaring active region is behind the limb, will it be possible to image faint HXR sources (below $\sim 1 \%$ of the brightest source), but the bulk of the HXR emission will not be measured.

One way to overcome this difficulty is to compare HXR measurements from instruments on board spacecraft viewing the Sun from widely different angles. Indeed, using such stereoscopic observations, Kane et al. (1992) identified an impulsive $>25 \mathrm{keV}$ HXR source extending to coronal altitudes $\approx 2 \times 10^{5} \mathrm{~km}$ above the photosphere. At $\sim 100 \mathrm{keV}$, the HXR emission from this coronal source was found to be $\approx 10^{-3}$ of the total flare HXR emission. Stereoscopic observations also contain information about the vertical structure of the HXR emitting volume (Kane et al. 1982) and about the directivity of the HXR emission (Kane 1987; Kane et al. 1998).

This paper reports on stereoscopic observations of the 1991 June $30 \mathrm{HXR} / \mathrm{GR}$ flare at $\sim 0255 \mathrm{UT}$ obtained by Ulysses/GRB and $C G R O / \mathrm{BATSE}$ (Sect. 2). The HXR emission from this flare was in full view of GRB, located $135^{\circ}$ east of the Sun-Earth line, but was partially occulted for BATSE which viewed the flare from the Earth (Sects. 3.1, 3.2). While the 30 June 1991 was one of the giant flares detected by GRB (Kane et al. 1995), for BATSE it appeared as a moderate electron-dominated event, and it was also detected up to $100 \mathrm{MeV}$ by PHEBUS (Vilmer et al. 1999). In this study, the combined GRB and BATSE HXR observations are used to determine the time evolution of: (i) the photon spectrum measured by BATSE, and the ratio of the partially occulted HXR emission seen by BATSE to the total HXR emission seen by GRB (Sects. 3.3, 3.4). The results are used to determine the spatial distribution of the HXR emitting source and its time evolution in the course of the event (Sect. 4). Conclusions are presented in Sect. 5.

\section{Instrumentation}

The Ulysses/GRB instrument (Hurley et al. 1992) consists of two $\mathrm{CsI}(\mathrm{Tl})$ scintillators, each with an effective area of $\sim 20 \mathrm{~cm}^{2}$. The lower energy threshold at which the detector is $10 \%$ efficient is $\sim 11 \mathrm{keV}$. During the 1991 June 30 hard Xray flare, Ulysses was located $135^{\circ}$ east of the Earth-Sun line at a distance of 3.48 AU from the Sun. Ulysses was in a solar proton event, and the background count rate of the GRB detector was about twice its normal value. Under these conditions, the experiment triggers constantly on particle fluxes, and therefore could not trigger on the solar HXR event. Since energy spectra are only recorded in triggered mode, the only data we have for the 1991 June 30 flare are $>28 \mathrm{keV}$ integral rates $\left(C_{28}^{\text {Uly }}\right)$ with a time resolution of $0.5 \mathrm{~s}$. In the following $C_{28}^{\mathrm{Uly}}$ includes appropriate corrections for dead time effects and counter overflow and is normalized to $1 \mathrm{AU}$.

The CGRO/BATSE instrument is described in Pendleton et al. (1995). The data used here consist of 16-channel spectra in the $\sim 20 \mathrm{keV}-8 \mathrm{MeV}$ energy range, obtained with the four Sunward large-area detectors (LADs) at a time resolution of $2.048 \mathrm{~s}$. During the 1991 June 30 flare, these data were recorded until 02:57 UT, when the Sun became Earth-occulted. In order to prevent pulse pile-up at the peak of the flare, the analysis was not performed on data from the detector whose axis was closest to the solar direction (LAD0), but rather from the next closest (LAD1) (Park et al. 1997; Vilmer et al. 1999). However, at the beginning of the event, when the count rates were low, LAD0 has also been used for spectral analysis to improve the statistics.

\section{Observations}

\subsection{Locations of the spacecraft relative to the flare}

Kane et al. (1995) reported that the 1991 June 30 HXR event was most likely associated with an optical flare located behind the east limb of the Sun. A detailed analysis of the various active regions crossing the east limb shortly after June 30 led to the estimate that the flare could have occurred anywhere between $2^{\circ}$ and $12^{\circ}$ behind the east limb in NOAA active region AR 6703 (see Vilmer et al. 1999). Figure 1 shows the ecliptic-plane projections of the longitude of AR 6703 and of the Ulysses and CGRO viewing directions. While the flare HXR emission was in full view of Ulysses, which was located $135^{\circ}$ east of the Sun-Earth line at a distance of $3.48 \mathrm{AU}$ from the Sun, HXR emission was also detected by $C G R O$.

\subsection{Occulting height for BATSE}

A flare site somewhere between $2^{\circ}$ and $12^{\circ}$ beyond the east limb corresponds to occulting heights in the range of $300-10^{4} \mathrm{~km}$, respectively, above the photosphere for BATSE. For giant flare, associated with optical flares on the disk or close to the limb, the soft X-ray (SXR) emission detected by the GOES detectors is very large (class $\mathrm{X}$ flares) and saturates the detectors for some events (see Kane et al. 1995). In contrast Fig. 2a shows that only a moderate SXR burst (M5.0) was observed for the 1991 June 30 event. Although, the SXR emission started to increase at $\sim 0240 \mathrm{UT}$, its main rise occurred at 0256:20 UT, about one minute after the onset of the GRB HXR burst (see Figs. 2a,b). Such a SXR-HXR timing is unusual. Generally, the SXR emission rises at the same time as or slightly before the HXR burst. The above remarks indicate that, during the main phase of the HXR flare ( 0255:20-0257 UT), 


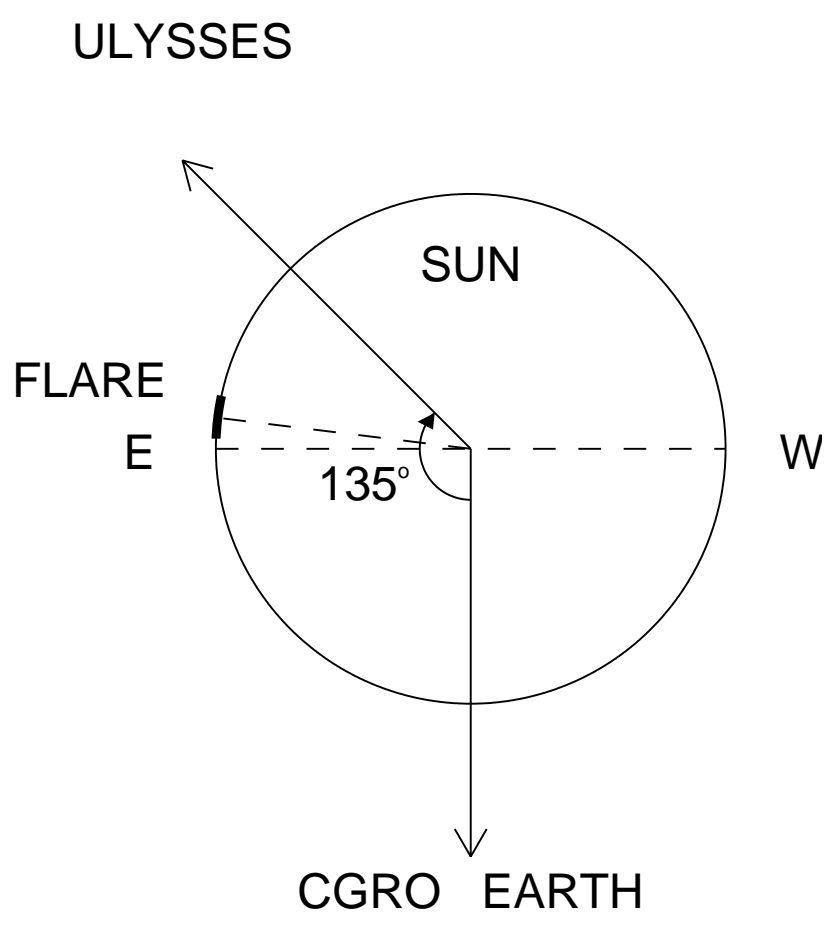

Fig. 1. Ecliptic-plane projection of the flare geometry. The longitude extent of the flaring active region (AR 6703) is indicated by the thick line on the solar disk. The arrows show the directions to Ulysses and $C G R O$.

a large fraction of the SXR emission was most probably occulted for a near-Earth orbiting detector. Noting that SXR imaging observations of impulsive flares show that the major fraction of the SXR emission is produced within loops with typical sizes of a few times $10^{4} \mathrm{~km}$, we conclude that the occulting height for BATSE is likely to be close or even larger than the upper limit $\left(10^{4} \mathrm{~km}\right.$ above the photosphere) inferred in Vilmer et al. (1999).

\subsection{Hard X-ray time histories}

The time histories of the HXR count rates measured by BATSE (LAD1) in several energy channels spread between 33 and $225 \mathrm{keV}$, and of $C_{28}^{\mathrm{Uly}}$ (normalized to $1 \mathrm{AU}$ ) are displayed in Fig. 3. The vertical dashed lines delineate three selected time intervals, labelled I to III. Figure 3 shows that the time profile of $C_{28}^{\text {Uly }}$ and of the 33-40 keV BATSE rate differ markedly although they are both produced by incident photons in similar energy ranges. Indeed:

- The event onset time, defined as the time when the rate increase starts to be more than 3-5 $\sigma$ above the background, has been estimated for the different BATSE energy channels and for $C_{28}^{\mathrm{Uly}}$. In order to have similar time resolutions for both instruments $C_{28}^{\mathrm{Uly}}$ has been accumulated over $2 \mathrm{~s}$. The onset of $C_{28}^{\text {Uly }}$ (marked by an arrow in Fig. 3) is found at $0255: 37 \mathrm{UT} \pm 2 \mathrm{~s}$, that is $\sim 18 \mathrm{~s}$ after the onset of the 33-40 keV BATSE rate (beginning of time interval I at 0255:19 UT). This does not necessarily indicate that the onset of the HXR emission detected at Ulysses is delayed with respect to that detected at $C G R O$ but, this rather reflects
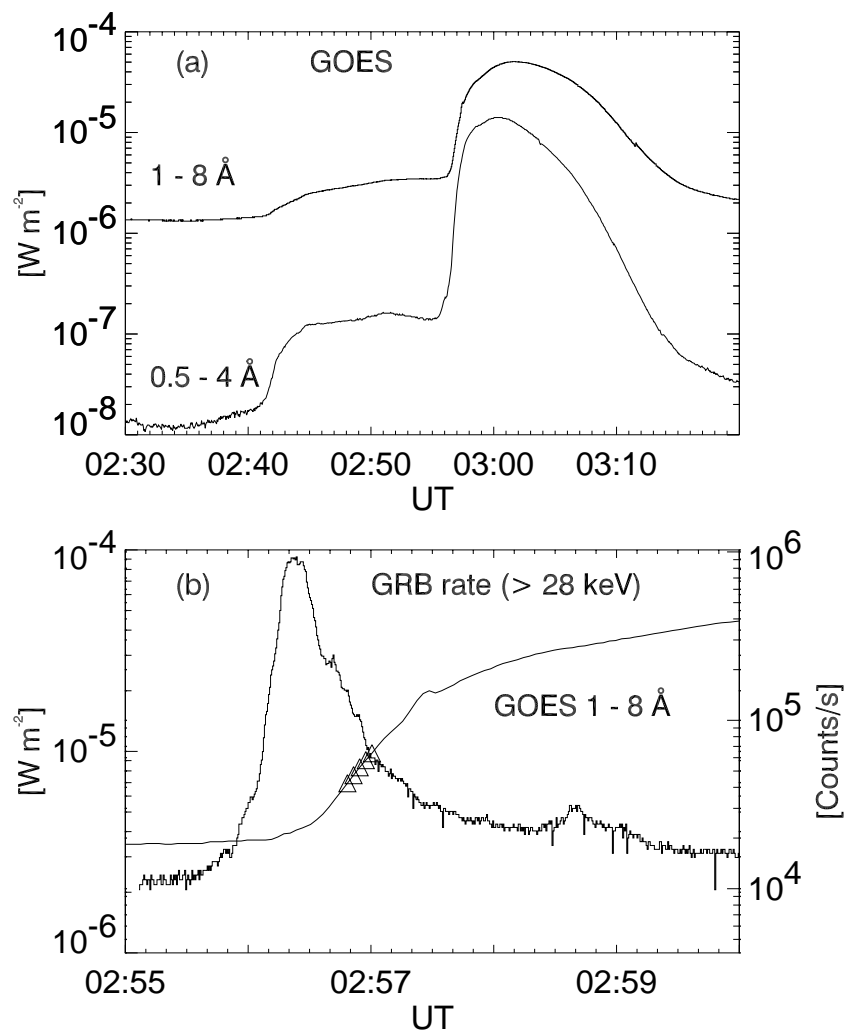

Fig. 2. a) Time evolution of the $1-8 \AA$ and $0.5-4 \AA$ SXR flux detected by the GOES detectors during the 1991 June 30 flare; b) The time profile of the $>28 \mathrm{keV}$ rate detected by GRB on Ulysses compared with that of the 1-8 A SXR flux where the triangles corresponds to interpolated values due to data gaps.

that GRB is less sensitive and farther away from the Sun than BATSE. Indeed, the average of $C_{28}^{\text {Uly }}$ over the time period 0255:19-0255:37 UT leads to a significant mean rate increase of $\sim 4 \sigma$ above the background. In the following, we thus consider, that the BATSE $33-40 \mathrm{keV}$ rate and $C_{28}^{\text {Uly }}$ have similar onsets.

- The main $C_{28}^{\text {Uly }}$ rise (beginning of time interval II at 0255:54 UT) is nearly simultaneous with the onset of the BATSE 158-225 keV HXR emission. Such a sudden rise is not reflected in the BATSE time profiles at energies below $60-70 \mathrm{keV}$.

- At a time resolution of $2 \mathrm{~s}$, the maximum of $C_{28}^{\mathrm{Uly}}$ occurs at 0256:22 UT $\pm 2 \mathrm{~s}$ (arrow in Fig. 3) in near coincidence with the BATSE maxima above $\sim 160 \mathrm{keV}$. However below $\sim 100 \mathrm{keV}$ the BATSE rate exhibits a broad maximum at 0256:30 UT $\pm 2 \mathrm{~s}$ (arrow in Fig. 3), i.e. $8 \mathrm{~s}$ later than the maximum of $C_{28}^{\mathrm{Uly}}$.

In summary, from the main rise of the event (beginning of time interval II) till the end of the analysed period (0257 UT), there is a closer similarity between the time evolutions of $C_{28}^{\mathrm{Uly}}$ and of the HXR emission seen by BATSE above $\sim 70 \mathrm{keV}$ than between $C_{28}^{\text {Uly }}$ and the HXR emission seen by BATSE at lower photon energies. 


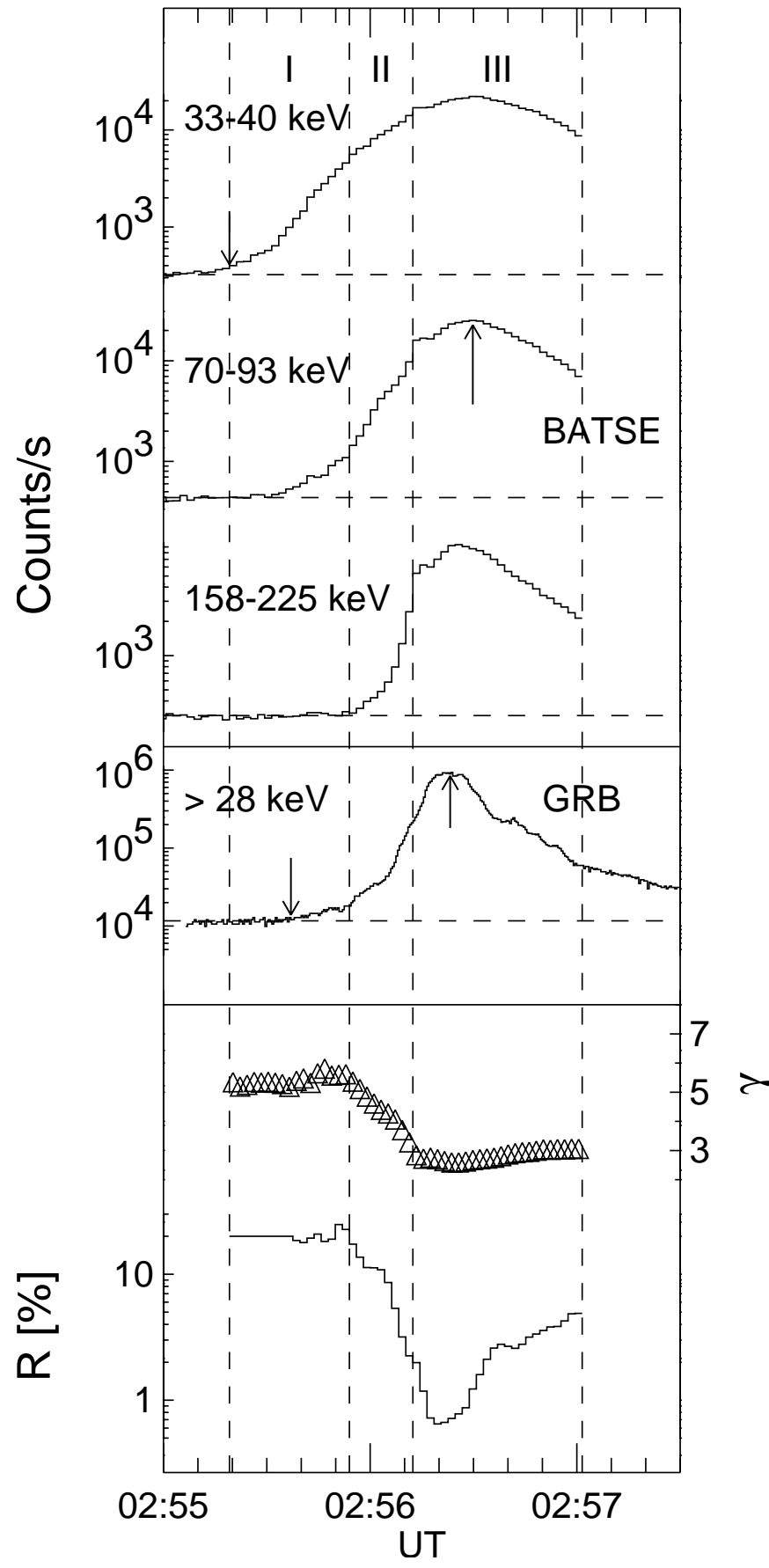

Fig. 3. The time evolution of: (i) the HXR rate observed by BATSE in three energy bands (time resolution $2.048 \mathrm{~s}$ ) and by GRB above $28 \mathrm{keV}$ normalized to $1 \mathrm{AU}\left(C_{28}^{\mathrm{Uly}}\right.$, time resolution $0.5 \mathrm{~s}$ ); (ii) the spectral index $\gamma$ of the photon spectrum inferred from BATSE data, and (iii) the ratio $R$ of the partially occulted $C_{28}^{\text {Earth }}$ to the unocculted $C_{28}^{\text {Uly }}>28 \mathrm{keV}$ HXR emission. The horizontal dashed lines indicate the background levels. The arrows mark the onsets of the $33-40 \mathrm{keV}$ BATSE rate and of $C_{28}^{\mathrm{Uly}}$ and the time of maximum of the 70-93 keV BATSE rate and of $C_{28}^{\mathrm{Uly}}$.

\subsection{Predicted $>28 \mathrm{keV}$ GRB rate at the Earth's orbit}

In the absence of GRB spectral data, we used BATSE spectral observations to predict the $>28 \mathrm{keV}$ rate $\left(C_{28}^{\text {Earth }}\right)$ that GRB would have measured if it was onboard CGRO. The following procedure was adopted:

- Each of the fifty count-rate spectra, recorded by BATSE from 0255:19 UT (onset of the 33-40 keV BATSE channel) to $0257 \mathrm{UT}$ (start of BATSE night), has been fitted to an incident power-law photon spectrum $\left(A(\mathrm{~h} v / 50)^{-\gamma}\right)$ in the 33-225 keV energy range. $\mathrm{h} v$ is the photon energy in $\mathrm{keV}$, $A$ (photons $\mathrm{cm}^{-2} \mathrm{keV}^{-1} \mathrm{~s}^{-1}$ ) the photon flux at $50 \mathrm{keV}$, and $\gamma$ the spectral index.

- For each $2.048 \mathrm{~s}$ interval, the GRB rate spectrum is estimated by convolving the photon spectrum determined from BATSE with the GRB response function. $C_{28}^{\text {Earth }}$ is then obtained by energy integration above $28 \mathrm{keV}$.

The above procedure assumes that thermal photons do not significantly contribute to the BATSE rate, and hence not to $C_{28}^{\text {Earth }}$ either. This assumption is supported by the fact that the 1-8 $\AA$ soft X-ray flux measured by GOES in Earth orbit is weak during the analyzed period of time (see Sect. 3.2). For such a low level of soft X-ray emission the contribution of thermal photons to the $\mathrm{X}$-ray emission above $28 \mathrm{keV}$ is negligible. Consequently, the accuracy of the $C_{28}^{\text {Earth }}$ is essentially determined by the reliability of the photon spectrum fitted to the BATSE data. The largest uncertainties in $C_{28}^{\text {Earth }}$ are thus those made during time interval I where the BATSE excess rates above 50 to $70 \mathrm{keV}$ are low (see Fig. 3). In order to estimate such an uncertainty, $C_{28}^{\text {Earth }}$ has been computed as above, during interval I, for each of the four sunward LADs (LAD0 is not affected by pulse pile-up during this time interval). The resulting four sets of $C_{28}^{\text {Earth }}$ values are in good agreement with a maximum relative spread of $30 \%$ at the beginning of interval I. We thus consider that the relative uncertainty in $C_{28}^{\text {Earth is no more }}$ than $30 \%$ for the whole of the event. The percentage of the occulted to unocculted $>28 \mathrm{keV}$ rate is given by:

$R=C_{28}^{\mathrm{Earth}} / C_{28}^{\mathrm{Uly}} \times 100$.

From 0255:19 UT to 0255:37 UT $R$ has been taken as the ratio of the average of $C_{28}^{\text {Earth }}$ to the average of $C_{28}^{\mathrm{Uly}}$. The statistical uncertainty on $C_{28}^{\mathrm{Uly}}$ is negligible compared to that made in estimating $C_{28}^{\text {Earth }}$, except between 0255:19 UT and 0255:37 UT, where the mean value of $C_{28}^{\mathrm{Uly}}$ is only $4 \sigma$ above the background. The uncertainty in $R$ is thus taken as $\sim 30 \%$ and somewhat larger at the beginning of the event.

The two bottom curves in Fig. 3 show the time evolutions of $\gamma$ and $R$. The time evolution of $\gamma$ led us to define the three intervals of time marked in Fig. 3. At the beginning of the event (interval I) $\gamma \sim 5$.4. It remains roughly constant and then decreases to $\sim 2.6$ during interval II. It remains at a value between 2.6 and 3.0 till the end of BATSE observations (interval III). As expected, $R$ is less than unity during the whole event, so that only a fraction of the total $>28 \mathrm{keV}$ HXR emission detected at Ulysses is observed in Earth orbit. However, this fraction varies greatly in the course of the event: $R$ decreases from $\sim 20 \%$ at the beginning of the event to less than $1 \%$ around the event maximum and increases to $\sim 5 \%$ in the event decay phase. Such a variation of $R$ is somewhat similar to that 
reported by Kane et al. (1982) from stereoscopic observations made during the impulsive phase of a different HXR flare. But in that flare, $R$ varied by less than a factor of 2 , whereas it varies by about a factor of 30 in the present case.

\section{Discussion}

Due to the lack of imaging HXR observations and of GRB spectral data, some assumptions have to be made in order to interpret the time evolution of $\gamma$ and $R$. In the following, we consider that:

(i) The major fraction of the occulted HXR emission $C_{28}^{\text {occ }}=$ $C_{28}^{\mathrm{Uly}}-C_{28}^{\text {Earth }}$ is produced by thick-target interactions of electrons (e.g. Brown 1971) precipitating at the chromospheric footpoints of HXR emitting loops. This is consistent with imaging observations of impulsive flares briefly outlined in Sect. 1.

(ii) The bulk of the unocculted HXR emission $\left(C_{28}^{\text {Earth }}\right)$ is produced in the coronal part of HXR emitting loops above the occulting height $\left(\gtrsim 10^{4} \mathrm{~km}\right)$, although a small fraction of it may originate from remote footpoints on the visible disk (see Sect. 4.3).

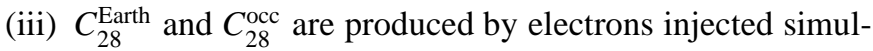
taneously at all energies, with the same spectrum, and from the same acceleration/injection region(s). The gross similarity between the time evolution of $C_{28}^{\text {Uly }}$ and of the BATSE rates (in particular above $70 \mathrm{keV}$ see, Sect. 3.3) supports this assumption.

(iv) Electron acceleration/injection regions are located in the corona at typical heights of a few times $10^{4} \mathrm{~km}$, close to the apex of SXR loops (e.g., Masuda et al. 1994) or at interaction regions between loops or loop systems of different spatial scales, typically $\sim 10^{4}-10^{5} \mathrm{~km}$ (e.g., Hanaoka 1997; Nishio et al. 1997; Manoharan et al. 1996). The ambient density of the acceleration/injection region was found to range typically from $\sim 10^{9} \mathrm{~cm}^{-3}$ to $\sim 10^{11} \mathrm{~cm}^{-3}$ (e.g., Klein 1995; Vilmer \& Trottet 1997, and references therein).

Given the above assumptions, the implications of the behaviour of $\gamma$ and $R$ for the spatial structure of the $>28 \mathrm{keV} \mathrm{HXR}$ source are qualitatively discussed below.

\subsection{Time evolution of $\gamma$}

Whereas $\gamma$ does not vary significantly during interval $\mathrm{I}$, it decreases from a value of $\sim 5.4$ to $\sim 2.6$ by the end of interval II. Figure 3 shows that the start of the progressive hardening of the photon spectrum of the partially occulted HXR emission occurs in near coincidence with (i) a marked change of the slope in the rise of $C_{28}^{\text {Uly }}$ and of the 70-93 keV BATSE count rate, and with (ii) the onset of the $\gtrsim 150 \mathrm{keV}$ HXR emission detected by BATSE. Points (i) and (ii) constitute signatures of the onset of a new injection of electrons with a harder spectrum than electrons injected during interval I. In agreement with this, Vilmer et al. (1999) reported that microwave emission at 17 and $35 \mathrm{GHz}$, from electrons with energies ranging from a few hundred $\mathrm{keV}$ to a few $\mathrm{MeV}$, started to be detected by the Nobeyama radio instruments $\sim 10 \mathrm{~s}$ after the beginning of interval II. The hardening with time of the photon spectrum measured by BATSE is thus the consequence of the overlap between the HXR emission produced during the decay of the soft electron injection, and the HXR emission produced during the initial rise of the hard injection. During interval III, $\gamma$ remains nearly constant between $\sim 2.6$ and $\sim 3.0$. This indicates that $C_{28}^{\text {Earth }}$ is produced by the hard population of electrons till the end of BATSE observations.

\subsection{Time evolution of $R$}

During interval I (soft electron injection), the nearly constant values of $R \sim 20 \%$ and $\gamma \sim 5.4$ (Fig. 3) provide evidence that there is a well-defined relationship, i.e. $C_{28}^{\text {Earth }} \approx 0.2 \times C_{28}^{\mathrm{Uly}}$, between $C_{28}^{\text {Earth }}$ and $C_{28}^{\mathrm{Uly}}$. This indicates that the physical parameters (e.g. size, ambient density) of the HXR source do not change substantially during interval I. A possible interpretation is that unocculted emission, $C_{28}^{\text {Earth }}$, was produced in a loop-top source and that occulted emission, $C_{28}^{\text {occ }}=C_{28}^{\mathrm{Uly}}-C_{28}^{\text {Earth }} \approx$ $0.8 \times C_{28}^{\text {Uly }}$, originated from the footpoints of the HXR emitting loop. Indeed, several characteristics of the X-ray emission measured during interval I are consistent with those obtained from Yohkoh/HXT imaging of a few, close-to-the limb flares exhibiting coronal HXR sources (Masuda et al. 1994, 1995):

- Coronal HXR sources detected by HXT are located typically $1-2 \times 10^{4} \mathrm{~km}$ above the photosphere, and about $7 \times$ $10^{3} \mathrm{~km}$ above flaring SXR loops (e.g. Aschwanden 2002, and references therein). This is fully consistent with the lack of an SXR increase at the beginning of the 1991 June 30 flare and with the inferred occulting height for BATSE (see Sect. 3.2 and Fig. 2).

- For the 30 June 1991 event, $C_{28}^{\text {occ }} / C_{28}^{\text {Earth }}=(100 / R)-1=$ $4 \pm 1.7$. This is comparable with the ratio $(\sim 5)$ of the footpoint to loop-top emission obtained by Masuda et al. (1995) above $30 \mathrm{keV}$.

- The present value of $\gamma \sim 5.4$ is in the range of values ( 4-6) obtained by Masuda et al. (1995) for HXR coronal sources.

$R$ decreases progressively from $\sim 20 \%$ at the end of interval I to $\sim 0.7 \%$ at the event maximum, i.e. during the rise of the hard injection of electrons. This indicates that the structure of the HXR source evolves with time so that $C_{28}^{\text {Uly }}$ grows faster than $C_{28}^{\text {Earth }}$. Such an evolution is consistent with an upward and/or eastward extension of the HXR source detected during interval I. Indeed:

- On one hand, $C_{28}^{\text {Earth }}$, which originates in a coronal source of low density $\left(\$ 10^{9}-10^{10} \mathrm{~cm}^{-3}\right)$ is proportional to the source ambient density $N_{\mathrm{c}}$. On the other hand, $C_{28}^{\text {occ }}$ which is produced in a thick-target is independent of the source density. For a given electron injection, an upward expansion of the coronal source will cause $N_{\mathrm{c}}$, and thus $R$, to decrease with time.

- In the case of an extension of the HXR source beyond the limb, a growing fraction of the source is progressively 
occulted. As the occulting height increases with the distance to the limb, a fraction of the coronal HXR emission is progressively produced at higher altitudes i.e. at lower ambient densities. Both effects lead to a decrease of $R$.

The present data do not provide sufficient constraints to rule out either one of the above scenarios. However, an upward extension of the HXR source would likely reflect the destabilization of the whole magnetic structure in which energy release occurred. It is thus expected that some of the SXR emitting loops, which were occulted during interval I, will rise above the occulting height. This is not observed since the SXR emission does not increase before the event maximum (see Fig. 2). We thus conclude that the decrease of $R$ was predominently the result of the eastward extension of the HXR source. Although all flares are different, it is interesting to note that such an expansion in longitude of the HXR source has been imaged for one large flare (Masuda et al. 2001; Fletcher \& Hudson 2001). Furthermore, this is in line with the suggestion by Kane et al. (1995) that for giant flares, the energy release and acceleration processes may operate on much larger scales than that of active region loops. In any case the injection of the hard population of electrons coincides with an expansion of the HXR emitting volume. A similar behavior has been reported by Trottet et al. (1998) for one electron-dominated event associated with a disk flare.

During the decay of $C_{28}^{\text {Earth }}$ and $C_{28}^{\text {Uly }}, R$ increases again from $\sim 0.7 \%$ at the event maximum to $\sim 5 \%$ at the end of BATSE observations. There are, at least, two possibilities that could explain this increase of $R$ :

- The emission from loops with footpoints far behind the limb decays faster than that from loops with footpoints closer to the limb. This may, for example, reflect changes of the injection conditions so that the relative number of electrons injected in the latter loops increases with time.

- The ambient density of the unocculted HXR source grows with time. This may, for example, be the result of the evaporation of the chromospheric gas heated by precipitating electrons (e.g., Doschek 1990; Antonucci 1994), and/or of the emergence of post-flare loops above the occulting height.

The increase of the SXR emission after the HXR event maximum (see Fig. 2) suggests that the latter effect may account, at least partly, for the increase of $R$. Indeed, assuming an isothermal SXR source, we find that the emission measure increases by a factor of $\sim 4$ from the event maximum till the end of interval III.

\subsection{HXR source on the visible disk}

Vilmer et al. (1999) reported that from the middle of interval II till the end of BATSE observations a high-energy GR continuum was detected, by PHEBUS in Earth orbit, up to a few tens of $\mathrm{MeV}$ (even up to $100 \mathrm{MeV}$ around the event maximum). This GR emission was most likely produced by thick-target interactions on the visible disk for the following reasons (see Vilmer et al. 1999, for a more complete discussion):

- the spectral characteristics of this hard $\left(\gamma_{\mathrm{GR}} \approx 1.9 \pm 0.2\right) \mathrm{GR}$ continuum are similar to the ones of electron-dominated events associated with flares on the visible disk (Rieger et al. 1998; Trottet et al. 1998);

- the production of the GR emission in a thin-target coronal source would imply an unrealistically flat electron spectrum (power-law index $\sim 1.2-1.6$ ).

Unless the low energy cut-off of the hard population of GR producing electrons is unusually high (this has not been observed so far), it will also produce lower energy X-rays in the disk source. For $\gamma_{\mathrm{GR}} \approx 1.9 \pm 0.2$, this emission represents $\sim 10 \% \pm 5 \%$ of $C_{28}^{\text {Earth }}$. At the maximum of the event when $R \sim 0.7 \%$, this corresponds to $\sim 0.07 \%$ of $C_{28}^{\mathrm{Uly}}$ and to an energy deposition rate in the disk source of $\sim 10^{27} \mathrm{ergs} \mathrm{s}^{-1}$ above $28 \mathrm{keV}$. It is likely that the onset of the high energy GR continuum occurred at the beginning of interval II, i.e. at the onset of the injection of the hard population of electrons, but the flux was too low to be detected within the sensitivity of PHEBUS. Thus, the HXR source on the visible disk, was probably present from the beginning of interval II till the end of the present observations.

\section{Conclusion}

The GRB, BATSE and GOES observations of the flare on 1991 June 30 (0255 UT) have provided information on both the vertical and horizontal structure of the $>28 \mathrm{keV}$ HXR source. On one hand, this flare was one of the giant flares detected by GRB (Kane et al. 1995), which saw the entire HXR source. On the other hand, it was partially occulted for BATSE (occulting height $\gtrsim 10^{4} \mathrm{~km}$ ). Thus, BATSE observed only a small fraction, ranging from $\sim 20 \%$ at the event beginning down to $\sim 0.7 \%$ at the event maximum, of the total HXR emission measured by GRB in those time frames. HXR/GR emission was also detected up to $\sim 100 \mathrm{MeV}$ by PHEBUS in Earth orbit, corresponding to that of a weak electron-dominated event (Vilmer et al. 1999). The time evolution of the ratio of the occulted to the unocculted $>28 \mathrm{keV}$ emission, and of the spectral index of the photon spectrum of the occulted emission shows the following:

- At the event beginning (interval I), the HXR emission was produced by a soft population of electrons and the spatial structure of the HXR source does not substantially vary with time.

- About $30 \mathrm{~s}$ after the event onset (beginning of interval II), the HXR source started to expand when a new injection of electrons with a much harder spectrum occurred. This source expansion continued till the HXR event maximum. The lack of a significant increase of the SXR emission during this time frame favors an eastward expansion beyond the limb. This is in agreement with multiwavelength studies of GR line flares and electron-dominated events which show that, in the course of a flare, strong acceleration up to relativistic energies, is triggered when 
energy release occurs in large-scale magnetic structures (Chupp et al. 1993; Trottet et al. 1994, 1998; Rieger et al. 1999). This is also in line with the statement by Kane et al. (1995) that for giant flares, the energy release and acceleration processes may operate on much larger scales than that of active region loops.

- The detection of a high energy GR continuum, by PHEBUS in Earth orbit (Vilmer et al. 1999) provides strong evidence that at least $10 \%$ of the $>28 \mathrm{keV}$ HXR emission detected by BATSE, i.e. $0.07 \%$ of the total emission at the event maximum, originated from an on-the -disk source from the beginning of interval II till the end of BATSE observations.

In summary, the present results indicate that the energy release during large solar flares seems to be a complex process which evolves with time and involves large spatial scales. Simple loop models may thus be inadequate to explain such a flare process. Imaging observations of HXR/GR events associated with disk flares constitute, in principle, the most direct way to further investigate such complexity in space and time during large and even weaker flares. However, due to their intrinsic dynamic range (100:1 in the case of RHESSI), such observations may reveal HXR spatial distributions which are simpler than the actual ones. This emphasizes the potential interest of combining imaging and full disk measurements of HXR flares obtained under widely different viewing angles.

Acknowledgements. One of us (GT) wishes to thank E. L. Chupp for his valuable comments and suggestions. We are also indebted to the referee, B. Dennis, for his constructive criticisms. KH is grateful for Ulysses support under JPL Contract 958056. GOES data were provided by the Solar Data Analysis Center at NASA/GSFC.

\section{References}

Antonucci, E. 1994, Space Sci. Rev., 70, 149

Aschwanden, M. J. 2002, Space Sci. Rev., 101, 1

Brown, J. C. 1971, Sol. Phys., 18, 489

Chupp, E. L., Trottet, G., Marschhauser, H., et al. 1993, A\&A, 275, 602
Dingus, B. L., Sreekumar, P., Bertsch, D. L., et al. 1994, in HighEnergy Solar Phenomena - a New Era of Spacecraft Measurements, ed. J. M. Ryan, \& W. T. Vestrand, AIP Conf. Proc., 294, 177

Doschek, G. A. 1990, ApJS, 73, 117

Duijveman, A., Hoyng, P., \& Machado, M. E. 1982, Sol. Phys., 81, 137

Fletcher, L., \& Hudson, H. 2001, Sol. Phys., 204, 69

Gallagher, P. T., Dennis, B. R., Krucker, S., Schwartz, R. A., \& Tolbert, A. T. 2002, Sol. Phys., 210, 341

Hanaoka, Y. 1997, Sol. Phys., 173, 319

Hurford, G. J., Schmahl, E. J., Schwartz, R. A., et al. 2002, Sol. Phys., 210,61

Hurley, K., Sommer, M., Atteia, J.-L., et al. 1992, A\&AS, 92, 401

Kane, S. R. 1987, Sol. Phys., 113, 145

Kane, S. R., Fenimore, E. E., Klebesadel, R. W., \& Laros, J. G. 1982, ApJ, 254, L53

Kane, S. R., Hurley, K., McTiernan, J. M., et al. 1998, ApJ, 500, 1003

Kane, S. R., Hurley, K., McTiernan, J. M., et al. 1995, ApJ, 446, L47

Kane, S. R., McTiernan, J., Loran, J., et al. 1992, ApJ, 390, 687

Klein, K.-L. 1995, in Coronal Magnetic Energy Releases, ed. A. O. Benz, \& A. Krüger, LNP No. 444 (Springer), 55

Manoharan, P. K., van Driel-Gesztelyi, L., Pick, M., \& Demoulin, P. 1996, ApJ, 468, L73

Masuda, S., Kosugi, T., Hara, H., et al. 1995, PASJ, 47, 677

Masuda, S., Kosugi, T., Hara, H., Tsuneta, S., \& Ogawara, Y. 1994, Nature, 371, 495

Masuda, S., Kosugi, T., \& Hudson, H. S. 2001, Sol. Phys., 204, 55

Nakajima, H., Dennis, B. R., Hoyng, P., et al. 1985, ApJ, 288, 806

Nishio, M., Yaji, K., Kosugi, T., Nakajima, H., \& Sakurai, T. 1997, ApJ, 489, 976

Park, B. T., Petrosian, V., \& Schwartz, R. A. 1997, ApJ, 489, 358

Pendleton, G., Paciesas, W., Mallozzi, R., et al. 1995, Nucl. Instr. Meth. Phys. Res. A, 364, 567

Rieger, E., Gan, W. Q., \& Marschhäuser, H. 1998, Sol. Phys., 183, 123

Rieger, E., Treumann, R. A., \& Karlický, M. 1999, Sol. Phys., 187, 59

Sakao, T. 1994, Ph.D. Thesis

Sakao, T., Kosugi, T., Masuda, S., et al. 1992, PASJ, 44, L83

Trottet, G., Chupp, E. L., Marschhaeuser, H., et al. 1994, A\&A, 288, 647

Trottet, G., Vilmer, N., Barat, C., et al. 1998, A\&A, 334, 1099

Vilmer, N., \& Trottet, G. 1997, in Coronal Physics from Radio and Space Observations, ed. G. Trottet, LNP No. 483 (Springer), 28

Vilmer, N., Trottet, G., Barat, C., et al. 1999, A\&A, 342, 575 